\title{
Fatal Hypermagnesemia Caused by an Epsom Salt Enema: A Case Illustration
}

\author{
Nancy M. Tofil, MD; Kim W. Benner, PHARMD; Margaret K. Winkler, MD
}

South Med J. 2005;98(2):253-256. C2005 Lippincott Williams \& Wilkins

Posted 03/17/2005

\section{Abstract and Introduction}

\section{Abstract}

The authors describe a case of fatal hypermagnesemia caused by an Epsom salt enema. A 7-year-old male presented with cardiac arrest and was found to have a serum magnesium level of $41.2 \mathrm{mg} / \mathrm{dL}(33.9 \mathrm{mEq} / \mathrm{L})$ after having received an Epsom salt enema earlier that day. The medical history of Epsom salt, the common causes and symptoms of hypermagnesemia, and the treatment of hypermagnesemia are reviewed. The easy availability of magnesium, the subtle initial symptoms of hypermagnesemia, and the need for education about the toxicity of magnesium should be of interest to physicians.

\section{Introduction}

Hypermagnesemia can be fatal, especially if a delay in recognition occurs. Initial symptoms of hypermagnesemia, such as nausea, vomiting, and weakness, are nonspecific and can progress rapidly to respiratory depression, hypotension, cardiac arrest, and death. Treatment is directed toward reversing the toxic effects of magnesium, primarily in the cardiovascular and nervous systems, discontinuing any further magnesium administration, and diuresis to aid in the elimination of excess magnesium. Hypermagnesemia is most commonly seen in patients with renal insufficiency; however, it can also be seen in individuals with normal renal function, if enough magnesium is administered orally, intravenously, or rectally. ${ }^{[1,2]}$ Therefore, to stress the importance of early recognition and prevention of accidental magnesium toxicity, we present a patient with fatal hypermagnesemia from an Epsom salt enema. The Institutional Review Board at the University of Alabama at Birmingham reviewed and approved publication of this case report.

\section{Case Report}

A 7-year-old male with a history of constipation presented to an outside hospital with cardiopulmonary arrest. He had complained of abdominal cramping the morning of admission and was given an unknown amount of a tap water and Epsom salt enema by his family in an attempt to relieve his abdominal pain. The family reports that initially the child felt better but then became sleepy and went to lie down. His family checked on him approximately 10 minutes later, found him difficult to arouse, and immediately took him to the local emergency room. On arrival to the emergency department, he was found to be in asystole. Cardiopulmonary resuscitation (CPR) was begun immediately with the administration of epinephrine, atropine, and sodium bicarbonate. The patient was intubated, volume resuscitated, and a continuous epinephrine infusion was begun. The patient was transported to our institution for further treatment. Brief periods of a perfusing cardiac rhythm occurred during transport but repeatedly degenerated into asystole, necessitating CPR and further boluses of epinephrine.

On arrival at our institution, the patient was found to be in asystole. CPR was continued with additional ad-ministration of epinephrine, atropine, calcium, bicarbonate, and fluid resuscitation. Calcium was given because of a low ionized calcium level drawn immediately on arrival to our intensive care unit. This was the first dose of calcium that the patient had received. Physical examination revealed a well-grown 7-year-old who was unresponsive to stimuli, with fixed, dilated pupils. Endotracheal and nasogastric tubes were placed. His lungs were clear to auscultation, and his abdomen was distended. Initial venous blood gas revealed a mild respiratory acidosis and an ionized calcium of $0.63 \mathrm{mmol} / \mathrm{L}$ (normal, 1.1 to 1.3 ).

Resuscitation efforts continued, including the administration of repeat doses of calcium with subsequent normalization of the ionized calcium. However, even with aggressive, on-going resuscitation efforts, only brief periods of sinus bradycardia with PR interval prolongation were achieved, which repeatedly degenerated into asystole. Forty-two minutes after presenting to our institution and approximately 3 hours after presenting to the outside hospital, the patient was pronounced dead. Shortly after his death, the remainder of his laboratory tests were returned ( Table 1) , revealing a magnesium level of $41.2 \mathrm{mg} / \mathrm{dL}$.

\section{Discussion}


Epsom salt is essentially $100 \%$ magnesium sulfate. One tablespoon of Epsom salt contains approximately $35 \mathrm{~g}$ of magnesium sulfate, which is $3.4 \mathrm{~g}(287 \mathrm{mEq})$ of elemental magnesium. This is a considerable amount, considering that the recommended daily allowance for an adult is only 300 to $400 \mathrm{mg}$ per day. ${ }^{[4]}$ Since that time, Epsom salt has been used for numerous ailments including abdominal pain, constipation, sprains, muscle strains, hyaline membrane disease, and cerebral edema. ${ }^{[5-8]}$

The first report of death caused by an Epsom salt enema was published in 1943 by Fawcett and Gens. ${ }^{[9]}$ Before this report, Fraser ${ }^{[5]}$ reviewed the literature on the toxicity of Epsom salt from 1841 to 1909 and found 7 cases of magnesium toxicity from Epsom salt use, with 5 deaths. Magnesium enemas were used in the treatment of infants with hyaline membrane disease in the early 1970 s until they were found to have no benefit and resulted in deaths caused by hypermagnesemia. ${ }^{[7]}$ Magnesium enemas were also used in the management of elevated intracranial pressure in the 1920s, based on an incorrect theory that causing water loss in the rectum would result in fluid loss in the brain and therefore might lower the intracranial pressure. ${ }^{[6]}$ Again, this was found to have no benefit and resulted in deaths caused by hypermagnesemia. ${ }^{[1]}$

Magnesium is one of the most abundant cations and is the second most abundant intracellular cation found in the body. It serves as an important cofactor for most cellular metabolic and energy-related reactions involving phosphorus. Magnesium is involved in more than 300 chemical reactions in the body including glycolysis, oxidative phosphorylation, and the proper functioning of the Na-K-ATPase pump. In addition to these roles, magnesium competitively binds to calcium channels and hence acts as a natural calcium channel blocker. [10,11]

Hypermagnesemia is a potentially lethal condition that may not be as rare as is commonly thought. In 1990, Whang and Ryder [12] studied more than 1,000 patients admitted to a community hospital and found the incidence of hypermagnesemia to be $5.7 \%$, whereas only $12 \%$ of those were clinically suspected. Hypermagnesemia is usually found in patients with renal insufficiency who have a creatinine clearance of less than $30 \mathrm{~mL} / \mathrm{min}$. Other patients at risk for development of elevated serum magnesium levels are patients with excessive magnesium loads. Potential sources of excess magnesium intake include antacids, laxatives, cathartics, and parenteral administration. Other less common causes of hypermagnesemia include rhabdomyolysis, tumor lysis syndrome, adrenal insufficiency,

hyperparathyroidism, and hypothyroidism. ${ }^{[13]}$

Although magnesium is in numerous over-the-counter agents and has many therapeutic uses, there are infrequent reports of death caused by magnesium toxicity. For many centuries, magnesium has been used to relieve gastrointestinal symptoms, including abdominal pain and chronic constipation. In patients with normal renal function, most extra magnesium ingested can be completely and easily eliminated. However, there are times when caution should be taken, and cases exist in the literature which highlight the potential danger of magnesium-containing products. ${ }^{[2,14-19]}$

Oral magnesium has also been used in the past as a cathartic agent in conjunction with oral activated charcoal for poisonings and overdoses. There have been numerous reports of fatal and near-fatal cases involving hypermagnesemia either in patients receiving magnesium with unrecognized renal failure or in patients with normal renal function who received charcoal and magnesium for treatment of an overdose. ${ }^{[20-22]}$ Many physicians now advocate the use of sorbitol instead of magnesium as the cathartic agent of choice in poisonings. ${ }^{[20,22]}$

Hypermagnesemia mainly affects the cardiovascular and nervous systems and does so in a dose-dependent manner. The initial effects of hypermagnesemia are nonspecific and can occur at levels of 3 to $4 \mathrm{mEq} / \mathrm{L}$. ${ }^{[9]}$ These include nausea, vomiting, cutaneous vasodilation, and feeling hot all over. As the serum magnesium level increases to 4 to $6 \mathrm{mEq} / \mathrm{L}$, deep tendon reflexes are lost and electrocardiographic changes occur, including PR interval prolongation and QRS interval prolongation. Hypotension, bradycardia, and further conduction abnormalities can occur at serum magnesium levels of 6 to $10 \mathrm{mEq} / \mathrm{L}$. As the level continues to rise to 10 to $15 \mathrm{mEq} / \mathrm{L}$, complete heart block, respiratory depression, and coma can occur. Asystolic arrest is a frequent finding with magnesium levels of greater than 15 $\mathrm{mEq} / \mathrm{L}$. $[13,23-25]$

The initial treatment in any patient with suspected magnesium toxicity is to discontinue any further intake of magnesium. Calcium directly antagonizes the membrane effects of hypermagnesemia and can reverse respiratory depression, hypotension, and cardiac dysrhythmias. Therefore, intravenous calcium should be given if there is any cardiopulmonary or neurologic compromise. Adequate hydration is necessary to help eliminate the excess magnesium through diuresis. Diuretics may also be used to enhance the diuresis, and if severe toxicity is present, immediate dialysis can be life-saving. Hemodialysis should be considered on any patient who has severe toxicity and is not responding to less invasive measures.

Our patient had many of the reported symptoms of hypermagnesemia; however, because of the severity of his exposure, he presented in asystole. After significant resuscitation, he had transient development of sinus rhythm with prolonged PR interval. His laboratory tests revealed hypocalcemia, which is often seen in patients with hypermagnesemia. Our patient's magnesium level was toxic because of a large rectal dose administered with significant rectal absorption. The patient had a history of constipation and may have had an enlarged rectal vault, providing more surface area for absorption. We had concern about a possible perforation of the rectum. However, autopsy results did not show any perforation. Also, the autopsy did not reveal any other cause for his death.

\section{Conclusion}

Because of the possibility of toxicity, the use of Epsom salt enemas should be discouraged. Rectal absorption of magnesium can be significant and difficult to predict, leading to toxic levels, even in patients with normal renal function. ${ }^{[1,2]}$ Hypermagnesemia should be suspected as a possible cause in any acidotic and hypotensive patient who presents to the hospital. Although hypermagnesemia is rare, if recognized early, it is easily treatable. Future efforts should be directed toward educating pediatricians and intensivists about early 
recognition of magnesium toxicity. Parents should also be educated about the potential toxicity associated with such a seemingly benign therapy, as there are many safer treatments available for constipation and abdominal pain.

\section{Table 1. Laboratory data}

\section{Medscape@ www.medscape.com}

\section{Table. Laboratory data}

Venous blood gas

$\mathrm{pH}$

$\mathrm{PCO}_{2}$

53

Bicarbonate $(\mathrm{mmol} / \mathrm{L})$

22

Ionized calcium $(\mathrm{mmol} / \mathrm{L})$

(normal range, 1.1-1.3)

Chemistries

Sodium $(\mathrm{mmol} / \mathrm{L})$

Potassium $(\mathrm{mmol} / \mathrm{L})$

Chloride $(\mathrm{mmol} / \mathrm{L})$

Bicarbonate $(\mathrm{mmol} / \mathrm{L})$

Blood urea nitrogen $(\mathrm{mg} / \mathrm{dL})$

Creatinine $(\mathrm{mg} / \mathrm{dL})$

Phosphorus ( $\mathrm{mg} / \mathrm{dL}$ )

Calcium $(\mathrm{mg} / \mathrm{dL})$

Magnesium $(\mathrm{mg} / \mathrm{dL})$

Hematology studies

Hemoglobin $(\mathrm{g} / \mathrm{dL})$

White cell count (per mL)

Differential count (\%)

Neutrophils

Lymphocytes

Bands

Myelocytes

Metamyelocytes

Reactive lymphocytes

Platelet count (per mL)

Prothrombin time (s)

(normal range, 11.3-14.9)

Partial thromboplastin time (s)

(normal range, 22.7-36)

\section{References}

1. Stevens AR, Wolff HG. Magnesium intoxication. Arch Neurol Psychiatry 1950;63:749-759.

2. Brown AT, Campbell WA. Hazards of hypertonic magnesium enema therapy. Arch Dis Child 1978;53:920.

3. Vissers RJ, Purssell R. latrogenic magnesium overdose: two case reports. J Emerg Med 1996;14:187-191.

4. Dietary Reference Intakes for Calcium, Phosphorus, Magnesium, Vitamin D, and Fluoride. ed. F.a.N.B. Standing Committee on the Scientific Evaluation of Dietary Reference Intakes, Institute of Medicine. Washington, DC, National Academy Press, 1997.

5. Fraser C. Epsom salts as a poison, with a record of a case of unusual symptoms due to this drug. Lancet 1909;1:1174.

6. Fay T. The administration of hypertonic salt solutions for the relief of intracranial pressure. JAMA 1923;80:1445.

7. Outerbridge EW, Papageorgiou A, Stern L. Magnesium sulfate enema in a newborn: fatal systemic magnesium absorption. JAMA 1973;224:1392-1393. 
8. Pray S. Constipation, in Pray WS (ed): Nonprescription Product Therapeutics. 1st edition. Philadelphia, Lippincott, Williams \& Wilkins, 1999, pp 131-154.

9. Fawcett D, Gens J. Magnesium poisoning following an enema of Epsom salt solution. Cardiovascular Defects 1943;123:1028-1029.

10. Kleinschmidt K, Delaney K. Magnesium, in Ford (ed): Clinical Toxicology. 1st edition. Philadelphia, WB Saunders \& Company, 2001, pp 87-89.

11. Swain R, Kaplan-Machlis B. Magnesium for the next millennium. South Med J 1999;92:1040-1047.

12. Whang R, Ryder KW. Frequency of hypomagnesemia and hypermagnesemia: requested vs routine. JAMA 1990;263:3063-3064.

13. Rosen M, Gibbs MA, Wolfson AB, et al. Electrolyte disturbances, in Marx (ed): Rosen's Emergency Medicine: Concepts and Clinical Practice. 5th edition. St. Louis, Mosby Inc, 2002, 1738-1740.

14. Morton A, Bailie G. Severe hypermagnesemia after MgSO4 enemas. Br Med J 1985;291:516.

15. McGuire JK, Kulkarni M, Baden HP. Fatal hypermagnesemia in a child treated with megavitamin/megamineral therapy. Pediatrics 2000;105:1-3.

16. Brand JM, Greer FR. Hypermagnesemia and intestinal perforation following antacid administration in a premature infant. Pediatrics 1990;85:121-124.

17. Schelling JR. Fatal hypermagnesemia. Clin Nephrol 2000;53:61-65.

18. Ashton MR, Sutton D, Nielsen M. Severe magnesium toxicity after magnesium sulphate enema in a chronically constipated child. BMJ 1990;300:541.

19. Curry CE, Butler DM. Constipation, in Berardi RR, DeSimone EM, Newton GD, et al (eds): Handbook of Nonprescription Drugs. Washington, D.C., American Pharmaceutical Association, 2002, ed 13, pp 319-320.

20. Weber CA, Santiago RM. Hypermagnesemia: a potential complication during treatment of theophylline intoxication with oral activated charcoal and magnesium-containing cathartics. Chest 1989;95:56-59.

21. Gerard S, Hernandez C, Khayam-Bashi H. Extreme hypermagnesemia caused by an overdose of magnesium-containing cathartics. Ann Emerg Med 1988;17:728-731.

22. Smilkstein MJ, Smolinske SC, Kulig KW, et al. Severe hypermagnesemia due to multiple-dose cathartic therapy. West J Med, 1988;148:208-211.

23. Mordes JP, Swartz R, Arky RA. Extreme hypermagnesemia as a cause of refractory hypotension. Ann Intern Med 1975;83:657-658.

24. Mordes JP, Wacker WE. Excess magnesium. Pharmacol Rev 1977;29:273-300.

25. Nakayama T, Nakayama h, Miyamoto M, et al. Hemodynamic and electrocardiographic effects of magnesium sulfate in healthy dogs. J Vet Intern Med 1999;13:485-490.

\section{Sidebar: Key Points}

- Epsom salt is essentially $100 \%$ magnesium sulfate, and ingestion can result in hypermagnesemia.

- Hypermagnesemia should be in the differential diagnosis of a patient presenting as the result of unexplained cardiopulmonary arrest.

- Rapid identification and treatment of hypermagnesemia can be life saving.

- Epsom salt is easily available, and practitioners should be aware of its potential toxicity.

\section{Reprint Address}

Reprint requests to Dr. Nancy Tofil, Pediatric Critical Care, 1600 7th Avenue South, ACC Suite 504, Birmingham, AL 35233. E-mail: ntofil@peds.uab.edu

Nancy M. Tofil, MD , Kim W. Benner, PHARMD , and Margaret K. Winkler, MD , Division of Pediatric Critical Care Medicine, Department of Pediatrics, the University of Alabama at Birmingham, Birmingham, AL, and Samford University McWhorter School of Pharmacy, Birmingham, AL

Disclosure: No author has any commercial outside interest in any drug, device, or equipment mentioned in the submitted article 